\title{
Outcomes Related to Electrolyte Imbalance in Patients Undergoing Chemotherapy; an Observational Study Focusing on Cancer Patients
}

\author{
Badria Munir ${ }^{1}$, Syed Mushhood Ali ${ }^{2}$, Reena Kumari ${ }^{3}$, Hobab Aslam ${ }^{4}$, Uzma Rehman $^{5}$, Amna Arshad ${ }^{6}$, \\ Natasha Mustafa ${ }^{7}$ and Adnan Anwar ${ }^{8^{*}}$ \\ ${ }^{1}$ MD, Clinical Research Fellow, Harvard Medical School and Beth Israel Deaconess Medical Center, USA \\ ${ }^{2}$ Medical Student, Hamdard College of Medicine and Dentistry, Hamdard University \\ ${ }^{3}$ MBBS, Post graduate Trainee, Department of Oncology \\ ${ }^{4} \mathrm{MBBS}$, House Officer, Patel Hospital \\ ${ }^{5} B D S$, MSc, Dental Officer, Sindh Government \\ ${ }^{6} \mathrm{MBBS}$, Hamdard college of Medicine and dentistry \\ ${ }^{7}$ MBBS, Lecturer,Department of Forensic Medicine, Al-Tibri Medical college Karachi \\ ${ }^{8}$ MBBS, M. Phil,Assistant Professor, Department of Physiology, Al-Tibri Medical College, Karachi
}

Received: May 22, 2019; Accepted: May 27, 2019; Published: May 29, 2019

*Corresponding author: Dr. Adnan Anwar, MBBS, M.Phil, Assistant Professor Department of Physiology Al Tibri Medical College Karachi, E-mail: anwaradnan32@gmail.com

\begin{abstract}
Objectives: Multiple studies are conducted to establish the frequency of electrolyte imbalance outside Pakistan but there is little data available locally. This study was therefore intended to assess the electrolyte imbalance in cancer patients undergoing chemotherapy which can help to reduce the morbidity related to electrolyte imbalance by replacing them appropriately.

Methodology: An observational study using non-probability convenient sampling technique was conducted for a year from April 2016 to March 2017 at the Oncology Department of Jinnah Post graduate Medical Center, Karachi after taking the ethical approval from the hospital's Ethical Review Committee. An informed consent was taken from a total of 256 cases diagnosed with various cancers and on an ongoing chemotherapeutic regimen before including them in the study. Age, height, weight, gender, body surface area, type of cancer, chemotherapy protocol, number of days on chemotherapy, electrolyte levels before and after therapy were the variables included. Data was analyzed using the SPSS 20 version. Descriptive statistics of demographic variables was presented as mean, standard deviation and frequency in percentages.

Results: 256 patients were included in the study. The mean age of patients was $43.21 \pm 12.85$ years. $52.7 \%$ of the patients showed a reduction in the level of sodium after treatment, followed by $52.2 \%$ of the patients showing a reduction in the level of potassium after treatment. $14.8 \%$ of the patients presented with reduced chloride levels, with $84.4 \%$ of the patients maintaining normal chloride levels before and after treatment. Furthermore, $87.1 \%$ of the patients, maintained normal magnesium levels, with $2.7 \%$ of the patients presenting with increased magnesium levels after therapy.

Conclusion: Our study predicted the significant reduction in the sodium and potassium levels in cancer patients on chemotherapeutic agents. However most of the patients maintained the normal levels of chloride and magnesium.
\end{abstract}

Key Words: Chemotherapy; Electrolyte Imbalance

\section{Introduction}

Cancer patients are generally challenged with a myriad of issues, electrolyte imbalance being one of them [1]. An ongoing chemotherapeutic regimen or paraneoplastic syndrome is also responsible for electrolyte imbalance [2]. A vigorous emergency plan and management is mandatory for timely correction of electrolyte imbalance in patients with malignant disorders in order to prevent mortality [3]. Hyponatremia, a decrease in the level of sodium, is the most widespread of electrolyte disorders that is associated with a malignant condition. In one of the recent studies, $14 \%$ of the overall patients with hyponatremia were suffering from cancer [4]. A well devised management plan and proper care is essential in preventing hyponatremia as in around half of 
the cases the hyponatremia developed was a hospital acquired, indicating a lack of effective care in the management of such cases in the hospital [5]. Tumor lysis syndrome, rhabdomylysis and renal injury are liable to cause hyperkalemia; an increase in the level of potassium [6]. Hyperkalemia is also attributable to adrenal insufficiency caused due to chemotherapeutic drugs and metastatic disease [7]. However, hypokalemia; a decrease in the level of potassium, is more frequently presented as compared to hyperkalemia in cancer patients and is documented as the second most common electrolyte disorder [8]. Loss of potassium via the gastrointestinal tract and renal tubular damage caused secondary to the usage of antibiotics such as amphotericin B and amino glycoside and certain chemotherapeutic drugs such as cisplatinand ifosfamide also contribute to hypokalemia [9]. Usage of diuretics in cancer patients, especially in ones with calcium levels of $11 \mathrm{mg} / \mathrm{dl}$ or above, plays a significant role in electrolyte imbalance causing hypokalemia and/or hyponatremia [10]. One of the case reports has also explored hypocalcaemia in patients suffering from cancer [11]. Calcium levels show a remarkable disturbance due to bone metastasis resulting in osteoblastic activity; however chemotherapy is usually a causative factor for hypomagnesaemia [12]. Electrolyte imbalance adds to the mortality burden in cancer patients [13].

Multiple studies are conducted to establish the frequency of electrolyte imbalance outside Pakistan but there is little data available locally. This study was therefore intended to assess the electrolyte imbalance in cancer patients undergoing chemotherapy which can help to reduce the morbidity related to electrolyte imbalance by replacing them appropriately.

\section{Methodology}

An observational study using non-probability convenient sampling technique was conducted for a year from April 2016 to March 2017 at the Oncology Department of Jinnah Post graduate Medical Center, Karachi after taking the ethical approval from the hospital's Ethical Review Committee. An informed consent was taken from a total of 256 cases diagnosed with various cancers and on an ongoing chemotherapeutic regimen before including them in the study. Patients with normal cardiac, renal, hepatic function and normal electrolyte were included in this study. Patients on total parental nutrition or with co morbid conditions, abnormal electrolyte values and/ or neurologic impairment were excluded from this study. Patients showing non-compliance during the study were also excluded. Single or combination of chemotherapy regimen that were used included taxotere, cisplatin and fluorouracil, oxaliplatin, leucovorin, adriamycin cyclophosphamide, gemcitabine, carboplatin, paclitaxel etoposide, daunorubicin, vincristine, prednisone, L-asparaginase, daunorubicin and cytarabine, hydroxydaunorubicin, oncovin, bleomycin, vinblastine and dacarbazine. Data was collected through performa and patients were followed for duration of three weeks after the completion of their chemotherapeutic regimen. Age, height, weight, gender, body surface area, type of cancer, chemotherapy protocol, number of days on chemotherapy, electrolyte levels before and after therapy were the variables included.

\section{Data Analysis}

Data was analyzed using the SPSS 20 version. Descriptive statistics of demographic variables was presented as mean, standard deviation and frequency in percentages. Correlation in independent variables including sodium, potassium, chloride, magnesium was studied. P-value of less than 0.05 was considered significant.

\section{Results}

256 patients were included in the study. The mean age of patients was $43.21 \pm 12.85$ years while weight was $56.02 \pm 11.25 \mathrm{~kg}$. The mean height was $158.38 \pm 10.55 \mathrm{~cm}$ and the mean body surface area was found to be $1.55 \pm 0.17 \mathrm{~m} 2$.. The days for chemotherapy were $5.98 \pm 2.36$ days. The most common group of cancer patient was acute myeloid leukemia, number of patients was about $58(23 \%), 35(14 \%)$ were of acute lymphoblastic leukemia and $30(12 \%)$ patients has cancer of ovary and lungs. In the sample size of 256 patients, sodium, chloride, potassium and magnesium levels were studied before and after the chemotherapeutic treatment. Patients with generally normal electrolyte levels before the study were included. $52.7 \%$ of the patients showed a reduction in the level of sodium after treatment, followed by $52.2 \%$ of the patients showing a reduction in the level of potassium after treatment. $14.8 \%$ of the patients presented with reduced chloride levels, with $84.4 \%$ of the patients maintaining normal chloride levels before and after treatment. Furthermore, $87.1 \%$ of the patients, maintained normal magnesium levels, with $2.7 \%$ of the patients presenting with increased magnesium levels after therapy.

\begin{tabular}{|c|c|c|}
\hline \multicolumn{2}{|c|}{ Table 1: Basic demographic variables } \\
\hline Variables (n=256) & Mean & Std. Deviation \\
\hline Age (years) & 43.21 & 12.85 \\
\hline Weight (kg) & 56.02 & 11.25 \\
\hline Height (cm) & 158.38 & 10.55 \\
\hline Body Surface area (m ${ }^{2}$ ) & 1.55 & 0.17 \\
\hline Chemotherapy (Days) & 5.98 & 2.36 \\
\hline
\end{tabular}

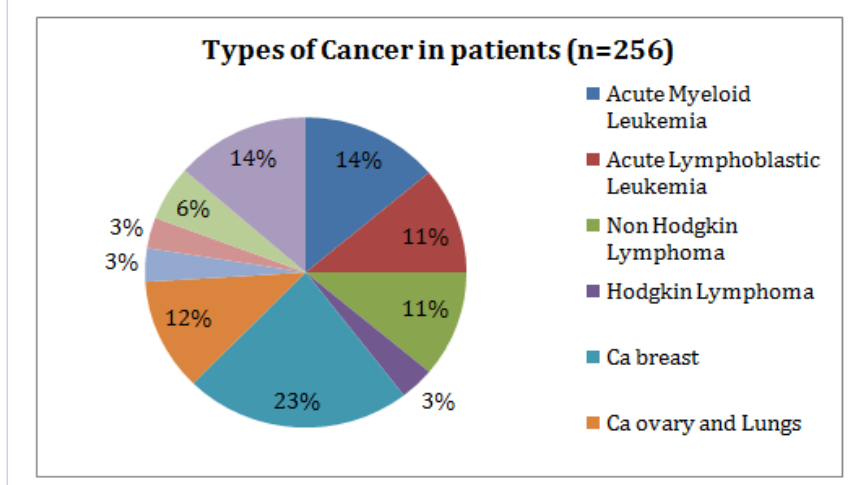

Figure 1: Type of cancer in patient under treatment $(n=256)$ 


\begin{tabular}{|c|c|c|c|c|c|c|}
\hline \multirow{2}{*}{\multicolumn{3}{|c|}{ Variables $(n=256)$}} & \multirow{2}{*}{\multicolumn{2}{|c|}{ Before Treatment }} & \multicolumn{2}{|c|}{ After Treatment } \\
\hline & & & & & & \\
\hline \multirow[b]{2}{*}{ Sodium } & Reduced & $<134.9$ & 2 & 0.8 & 135 & 52.7 \\
\hline & Normal & $135-145$ & 253 & 98.8 & 120 & 46.9 \\
\hline \multirow{3}{*}{ Chloride } & Reduced & $<96.9$ & 0 & 0 & 38 & 14.8 \\
\hline & Normal & $97-107$ & 254 & 99.2 & 216 & 84.4 \\
\hline & Increased & $>107.1$ & 2 & 0.8 & 2 & 0.8 \\
\hline \multirow{3}{*}{ Potassium } & Reduced & $<3.49$ & 2 & .8 & 108 & 52.2 \\
\hline & Normal & $35-5.0$ & 253 & 98.8 & 141 & 55.1 \\
\hline & Increased & $>5.01$ & 1 & 0.4 & 7 & 2.7 \\
\hline \multirow{3}{*}{ Magnesium } & Reduced & $<.15$ & 0 & 0 & 26 & 10.2 \\
\hline & Normal & $1.5-2.5$ & 255 & 99.6 & 223 & 87.1 \\
\hline & Increased & $>2.51$ & 1 & 0.4 & 7 & 2.7 \\
\hline
\end{tabular}

\section{Discussion}

The incidence of hyponatremia is the most common of electrolyte disorders faced by cancer patients and has increased from $4 \%$ to $47 \%[14,15]$. One of the studies shows that it is equivalent to the prevalence rates of hyponatremia 15\%-30\% reported for the patients in the general medicine wards [16]. A study conducted by Hutchison FN et alshows that lack of proper management during hospitalization is responsible for $14 \%$ of the cases of hyponateremia [17]. Whereas a separate study recorded that in $46-54 \%$ of the cases of hyponatremia, metabolic derangement is responsible [18]. These observations are similar to our study in which we also observed the reduction in the sodium levels in patients treated with chemotherapeutic agents. A study conducted by Alexandraki KI et al., shows that tumors which induce the secretion of ectopic (ACTH) such as SCLC, thyroid medullary carcinoma, thymus or bronchial carcinoid, or neuroendocrine tumours are liable to cause hypokalemia in cancer patients.In rare cases, tumors are found to fuel renal potassium wasting due to the activation of mineral-corticoid pathway leading to severe potassium loss requiring vigorous management and replacement [19]. Management of hypokalemia in cancer patients is the identical as that for the general patients. However, intravascular medication is considered superior to oral for oncological patients with complains of nausea and vomiting [20]. This finding is similar to our study in which $52 \%$ of the patients were suffering from hyperkalemia during chemotherapy.

One of the studies reported significant correlation in cancer patients on chemotherapy developing blood magnesium changes $\left(r^{2}=0.7455\right)$ [21]. The treatment of hypokalemia is largely dependent on the correction of hypomagnesaemia, if both are occurring simultaneously in a patient. The cause for this is the loss of potassium through Renal Medullar K1 channels (ROMK) in distal nephron tubular cells [22]. Hypomagnesaemia can occur due to decreased intake of magnesium or due to renal loss of magnesium. Distal nephron is the main site for the reabsorption of magnesium and unfortunately also a site exposed injurious side effects of chemotherapy [23].In one of the studies, 43 percent of the patients receiving platinum based chemotherapy have reported with hyponatremia [24].

The general conclusions found in the studies mentioned in this discussion show a wide range of electrolyte imbalances in cancer patients undergoing chemotherapy; a finding which is consistent with our study.Although, our research might not be invulnerable to selection and observer bias.

\section{Conclusion}

Our study observed a change in the electrolyte levels in patients after receiving chemotherapy. Electrolyte imbalances were observed mainly in the levels of sodium, potassium, magnesium and chloride during chemotherapy. Therefore, it is recommended that focus should be placed on devising a proper chemotherapeutic plan along with thorough management of electrolyte imbalances as it may help decrease mortality and morbidity in future.

\section{References}

1. Bowman BT. Electrolyte Disorders Associated with Cancer. Journal of Onco-Nephrology. 2017;1(1):30-35.

2. Nriagu J, Darroudi F, Shomar B. Health effects of desalinated water: Role of electrolyte disturbance in cancer development. Environ Res. 2016;150:191-204. doi: 10.1016/j.envres.2016.05.038

3. Kumar RV, Bhasker S. Health-care related supportive-care factors may be responsible for poorer survival of cancer patients in developing countries. Journal of Cancer Policy. 2015;5:31-47.

4. Allolio B, Annane D, Ball S, Bichet D, Decaux G, Fenske W, et al. Clinical practice guideline on diagnosis and treatment of hyponatraemia. Intensive Care Med. 2014;40:320-331. 
5. Moritz ML, Ayus JC. Maintenance intravenous fluids in acutely ill patients. N Engl J Med. 2015;373(14):1350-1360. doi: 10.1056/ NEJMra1412877

6. Lameire N, Van Biesen W, Vanholder R. Electrolyte disturbances and acute kidney injury in patients with cancer. Semin Nephrol. 2010;30(6):534-547. doi: 10.1016/j.semnephrol.2010.09.002

7. Carvalho F, Louro F, Zakout R. Adrenal Insufficiency in Metastatic Lung Cancer. World J Oncol. 2015;6(3):375-377. doi: 10.14740/wjon890w

8. Latcha S. Electrolyte Disorders in Cancer Patients. Onconephrology: Cancer, Chemotherapy and the Kidney. 2015;131-162.

9. Eun JN, Choi YD, Lee JH, Jeong YA, Yoon JH, Kim HK, et al. Severe Hypocalcemia in a Patient with RecurrentChondrosarcoma.Intern Med. 2017;56(14):1839-1842. doi: 10.2169/internalmedicine.56.7884

10. Fokkema MI, de Heide LJ, van Schelven WD, Hamdy NA. Severe hypocalcaemia associated with extensive osteoblastic metastases in a patient with prostate cancer. Neth J Med. 2005;63(1):34-37.

11. Eun JN, Choi YD, Lee JH, Jeong YA, Yoon JH, Kim HK, et al. Severe Hypocalcemiain a Patient with RecurrentChondrosarcoma. Intern Med. 2017;56(14):1839-1842. doi: 10.2169/internalmedicine.56.7884

12. Fokkema MI, de Heide LJ, van Schelven WD, Hamdy NA. Severe hypocalcaemia associated with extensive osteoblastic metastases in a patient with prostate cancer. Neth J Med. 2005;63(1):34-37.

13. Yadav Arvind, Khodke Prasad. Status of serum electrolytes in cancer patients. International Journal of Basic and Applied Medical Sciences. 2015;5(1):208-211.

14. Doshi SM, Shah P, Lei X, Lahoti A, Salahudeen AK. Hyponatremia in hospitalized cancer patients and its impact on clinical outcomes. Am J Kidney Dis. 2012;59(2):222-228. doi: 10.1053/j.ajkd.2011.08.029
15. Berghmans T, Paesmans M, Body JJ. A prospective study on hyponatraemia in medical cancer patients: epidemiology, aetiology and differential diagnosis. Support Care Cancer. 2000;8(3):192-197.

16. Verbalis JG, Goldsmith SR, Greenberg A, Korzelius C, Schrier RW, Sterns $\mathrm{RH}$, et al. Diagnosis, evaluation, and treatment of hyponatremia: expert panel recommendations. Am J Med. 2013;126(10 Suppl 1):S1-42. doi: 10.1016/j.amjmed.2013.07.006

17. Hutchison FN, Perez EA, Gandara DR, Lawrence HJ, Kaysen GA. Renal salt wasting in patients treated with cisplatin. Ann Intern Med. 1988;108(1):21-25.

18. Castillo JJ, Vincent M, Justice E. Diagnosis and management of hyponatremia in cancer patients. Oncologist. 2012;17(6):756-765. doi: 10.1634/theoncologist.2011-0400

19. Alexandraki KI, Grossman AB. The ectopic ACTH syndrome. Rev Endocr Metab Disord. 2010;11(2):117-126. doi: 10.1007/s11154010-9139-z

20. Unwin RJ, Luft FC, Shirley DG. Pathophysiology and management of hypokalemia: a clinical perspective. Nat Rev Nephrol. 2011;7(2):7584. doi: $10.1038 /$ nrneph.2010.175

21. Tsujii T, Ogaki T, Nakae K, Imai K, Kise D, Tada S, et al. Correlation between blood magnesium and calcium concentration in patients treated with an anti-EGFR antibody. Journal of pharmaceutical health care and sciences. 2016;2(1):23.

22. Huang CL, Kuo E. Mechanism of hypokalemia in magnesium deficiency. JASN 2007;18(10):2649-2652.

23. Groenestege WM, Thébault $S$, van der Wijst J, van den Berg D, Janssen R, Tejpar S, et al. Impaired basolateral sorting of pro-EGF causes isolated recessive renal hypomagnesemia. J Clin Invest. 2007;117(8):22602267.

24. Liamis G, Milionis H, Elisaf M. A review of drug-induced hyponatremia. Am JKidney Dis. 2008;52(1):144-153. doi:10.1053/j.ajkd.2008.03.004 